\title{
"From an Old Life to a New Life": Group Therapy for Cognitive Disorders and Affective Deficits in Adolescents Who Have Committed Sexual Offenses
}

\author{
Talia Etgar ${ }^{\mathrm{a}}$, Noam Neder ${ }^{\mathrm{b}}$
}

[a] Therapist and Head of Sex Offending Treatment Centers, ELEM, Tel Aviv, Israel. [b] Therapist, ELEM, Tel Aviv, Israel.

Sexual Offending: Theory, Research, and Prevention, 2020, Vol. 15(1), Article e3125,

https://doi.org/10.5964/sotrap.3125

Received: 2020-01-20 • Accepted: 2020-03-23 • Published (VoR): 2020-07-22

Handling Editor: Martin Rettenberger, Centre for Criminology (Kriminologische Zentralstelle - KrimZ), Wiesbaden, Germany

Corresponding Author: Talia Etgar, 4 Wiess st, Tel Aviv 69124, Israel. E-mail: Etgar_a@netvision.net.il

\begin{abstract}
The purpose of the current article is to shed light on the unique population of adolescents who have committed sexual offenses, suffering from cognitive disorders, by presenting the disorders and challenges that characterize these youngsters and discussing the issues that are relevant to the therapeutic process of sexual offenses. In our article we show the correlations and adaptations that we made to provide these boys with the most effective treatment possible, both to enable them to undergo an optimal process and to lower their risk level for recidivism.
\end{abstract}

\section{Keywords}

adolescents, sexual offending, group therapy, cognitive disorders, recidivism risk

\section{Highlights}

- Adolescents who have committed sexual offenses and who suffer from cognitive disorders are in higher risk and causes greater concern than other adolescents.

- A new approach is needed - one that integrates various working methods according to the characteristics of this population.

- Importance of the terms "new life" - and not "the new me" - and "old life". Understanding that change is a continuous process.

- Special attention to the therapists, who have different questions and doubts treating this population, which are similar to those of their patients. 
ELEM $^{1}$ has been working for many years in the diagnosis and treatment of adolescents who have committed sexual offenses and their families. The nonprofit organization's major efforts to focus on this population are intended to open a toolbox for therapists to help them cope with various issues arising from the field and deal with specific populations. The goal is to understand different social sectors, including their unique characteristics and the social codes that regulate their behaviors, and to find specific and appropriate responses for each one: "We believe that the same therapeutic principles that are an integral part of the treatment of adolescent who have committed sexual offenses must be processed in various ways through the therapeutic process, depending on the individual adolescent. In other words, the terms are not independent of each other, but are defined within the context of each adolescent" (Etgar \& Neder, 2008, p. 219). Among others, work with adolescents who have committed sexual offenses, is based on several basic principles, as well as on the mutual relationship between them: placing boundaries, and mainly the perception of the patient's boundaries, authoritative treatment and the use of a deterrent, as well as the "secret" and the confidentiality (pp. 221-222).

This article will attempt to highlight the treatment of adolescents who have committed sexual offenses who suffer from cognitive disorders. Though the literature on people with intellectual developmental disorder (IDD) who commit inappropriate sexual behavior has witnessed a surge in research in the past ten years, and despite the apparent overrepresentation of IDD among sexual offenders, empirical progress continues at a much slower rate than for that of sexual offenders without IDD (Craig, 2017). The knowledge and experience presented in this report are based on treatments provided at ELEM's various centers, as well as on the success acquired during group counseling sessions, as part of the program for group treatment for adolescents who have committed sexual offenses run by the Ministry of Welfare, the Youth Probation Service and the Youth Protection Authority. Details in the following examples have been changed to prevent any identification of the offenders and protect personal confidentiality.

For many years we met with adolescents (both in individual and group therapy) who had, in fact, never received an appropriate therapeutic response, had difficulty with individual treatments and were expelled, at one stage or another, from the therapy groups. For example, adolescents who, for reasons of their inability to think abstractly and focus concretely, could not understand what was said during therapy, and especially during processes of conceptualization. The process we witnessed often included withdrawal to their comfort zone (generally following years of frustration from the education system), to behavior that was infantile, shameless, or objecting and disturbing to the normal process of treatment, and mainly criticized boundaries, or alternatively, presented phobic, avoiding and withdrawn behavior. Therapists often reported the feeling that the accepted

1) "ELEM - Youth in Distress" was founded out of a sense of social responsibility for the fate of youth at-risk in Israel. 
methods of treating this population even bordered on the abuse of adolescents who could not understand what was going on during the therapy sessions. The situation encouraged us to find another therapeutic approach that could take the special difficulties of this population into account and provide a more appropriate, more precise and more suitable response, of course with the same therapeutic goals of stopping the abusive behavior, as well as building an improved sense of self-value and personal welfare, as to be described below.

We will start by saying that there was great deliberation on how to define this population: Lowered? Challenged? Having cognitive disorders? We believe that this debate is part of the general uncertainty on how to define sexually abusive adolescents (Etgar, 2012), but we will not discuss this in the current article, which will focus more on the therapeutic work with this specific population. We term these groups - groups of adolescents who have cognitive disorders and committed sexual offenses.

\section{Treatment of Sexually Abusive Adolescents}

The main treatment goals of sexually abusive minors, both children and adolescents, includes taking responsibility for the crime, amending errors in thought processes, training and improving their interpersonal and intimate relationships, awareness and empathy for the victim, preventing recurrence of the crime, controlling sexual arousal and strengthening family support systems. The main principles described in the literature are based, in general, on the behavioral-cognitive theory (Maletzky \& Steinhauser, 2002), the importance of a peer group (Brannon \& Troyer, 1995; Etgar, 1999), and the importance of a family system, mainly the parents (Schladale, 2006; Zankman \& Bonomo, 2004).

Marotta (2017) describes studies of programs designed to treat sexual offenders with intellectual and developmental disabilities (IDD; although not adolescents) and supports the importance of cognitive-behavioral treatments, that were the most commonly delivered treatment modality to sexual offenders with IDD. Other less common treatments were dialectical behavioral therapy, problem solving therapy, mindfulness, and relapse prevention.

Thibaut et al. (2016) - referring to adolescents with paraphilic disorders who are also sexual offenders or at-risk of sexual offending also mentioned treatment modalities stated that most of the published treatment programs have used cognitive behavioral interventions, family therapies and psychoeducational interventions. Psychological treatment is predicated in adolescents on the notion that sexually deviant behavior can be controlled by the offender, and that more adaptive behaviors can be learned.

One of the more well-known terms in this form of treatment is the Sexual Cycle. According to Lane (1991, pp. 103), the Sexual Cycle provides a theoretical framework that gives the adolescent offenders and the therapeutic team a chance to understand and examine the offending sexual behavior and develop methods of intervention to prevent 
the recurrence of this behavior. She adds that this Cycle combines and merges situations, thoughts, emotions and behaviors into one conceptual framework (Lane, 1991, pp. 115).

\section{Who Are the Adolescents Who Have Committed Sexual Offenses and Are Suffering From Cognitive Disorders?}

The population of special-ed students is defined as a population with lower developmental achievements than normatively expected. The students are defined as having cognitive, social, and emotional disorders. The disorders can be expressed in attention and concentration deficits, concrete and/or structured thought processes, limited egos and a low level of distinction (in self-awareness, building self-identity and recognizing differences in the other). Additional means of expression are dependency, difficulty to cope with doubt, vagueness and uncertainty, difficulty in understanding changes and social situations, and difficulty in judgment and assessing reality. Students in these schools tend to be classified also as impulsive, suffering from over-activeness and lack of control, inability to delay instant gratification, and more (Fisher-Caspi \& Vangruber-Neumann, 2010).

As stated, the "technical" definition of a male adolescent suffering from cognitive disorders, or an adolescent studying in a special-ed environment, does not explain the entire complexity of working with this population, especially in an area as difficult and sensitive as sexual offenses. We are talking about a population with low cognitive, and especially labial-verbal, abilities, generally resulting from organic development difficulties. We would like to emphasize that we are not talking about mental-developmental disorders (retardation) and it is important to remember that their emotional, psychological and social needs are identical to those within the general population. When these adolescents come to us for treatment, they are generally carrying on their shoulders experiences of anxieties, difficulties, frustrations, anger, failures, disappointments and pain from dealing with basic growing-up and development tasks. They also bear within themselves the disappointed and dissatisfied look on the faces of their parents and other significant adults (Fisher-Caspi \& Vangruber-Neumann, 2010).

The history of their mutual relationships with their environment is difficult and laden with bad influences. Over the years they were belittled and set in a fixed approach by those around them and this repressed them even more, which can be described as a vicious cycle. The same attitude that was constantly emphasized by the environment ("you don't understand," “you are a failure," "you don't know"), which they heard day and night, directly or indirectly, became an integral part of their personality, and some define and regard themselves as such. In other words, they have adopted an approach of "it's not even worth trying, because in any case I won't succeed." Their experiences 
throughout life are different and others do not perceive them or their needs as they truly are.

\section{What Is the Purpose of the Treatment?}

Before we describe the unique nature of treating this population in the context of sexual offenses, we are going to examine the actual purposes of the treatment. We will start by saying that the purposes of treatment for all populations are identical, with differences in the various methods of coping with diverse populations, which each affect the methods of treatment. As stated, the purpose of the treatment is to offer the adolescents who have committed sexual offenses, individual or group psychotherapeutic assistance, that is adapted to their abilities, in order to decrease their risk level to commit future sexual offenses. The criteria for lowering the risk level and defining treatment as successful are complex. This is in regard to all sexual offenders, and even more so to this social group. It is obvious that the main objective is to stop the offensive behavior.

Group therapy for adolescents who have committed sexual offenses, is based on accepting responsibility and teaching each adolescent the cognitive and emotional patterns that motivate their actions, including sexual offenses (the Sexual Cycle). Treatment is based on expanding the ability to make choices and correct errors in thought. A study conducted by Ofek (2006) enumerates several elements to ensure successful treatment of adolescent who have committed sexual offenses (pp. 92-93):

1. Stopping to perform sexual offenses.

2. Getting to know and understanding the Sexual Cycle from the adolescents' perspective. They know how to diagnose their own risk points, avoid these situations and cope using alternative methods. In addition, the adolescents identify the crime cycle not only in regard to sexual offenses, but from a much wider perspective of negative or offensive behaviors.

3. Understanding the extent of the physical, emotional and social offense on the victim. The adolescents identify with the victim's perspective and treat the victim as a human being and not as an object.

4. The adolescents manage to function on a daily basis outside of the group and continue to live within the community.

\section{Unique Nature of Treatment of Adolescents Who Have Committed Sexual Offenses Suffering From Cognitive Disorders}

The accepted therapeutic approach to sexual offenses committed by adolescents has numerous special reference points when dealing with this population. We will mention 
several of them. It is important to note that there are developmental distresses connected to problematic sexual behaviors among adolescents, including trauma, inability to communicate and dysfunctional families. These stresses are found also among sexual offenders with a lower intelligence (Lindsay et al., 2001; Thompson \& Brown, 1998), but there are certain areas that have greater effect on those with intelligence disorders, such as limited social development, social isolation, limited sexual education, lack of privacy, lack of opportunities to experience normative and appropriate sexual interactions, and difficulties in communication.

For example, the fact that the adolescents came for treatment, and the fact that they were punctual, is brought up in many cases. Generally, not coming or not coming on time are connected to fears of treatment, acting out for treatment, etc. But there is a difference with this population, or more precisely, there are additional factors: sometimes there is a misunderstanding, lack of reading the situation correctly, or difficulty in organization. Following are several examples: From the outset Moshe (not his real name) was willing to share personal thoughts that crossed his mind daily and he did not object to treatment. Even so, he did not come to meetings regularly, claiming he was "sick." He did not understand why he needed confirmation that he was sick; unlike Moshe, Baruch (not his real name) was very nervous and did not want to participate in the group. His solution was not to come at all. He needed several meetings with the counselors until they managed to explain to him the importance of the group session. Even these helped only for a short period of time. In other populations, one meeting would have generally been sufficient. Another example is Zacki (not his real name), who had difficulty planning his arrival (resulting from a severe disorder in planning and organization) and we needed to involve a parole officer, who taught him the importance of being punctual. The same applies to the fact that it is so clear to all, that if you are not coming, you notify those waiting for you.

\section{Cognitive Ability}

Working with adolescents who have committed sexual offenses is partially based on cognitive abilities. Later in the treatment process the patient learns about the Sexual Cycle (Lane, 1991) and works on his own cycle. As stated above, in order to understand the reasons for the events, the cause and effect, the social understanding and ability to make a choice, including what is "sex by consent," requires a certain level of cognitive and mentalization abilities. One of the important elements of this therapeutic work is dealing with "cognitive distortions" (Etgar, 1997, p. 47). This does not mean a thought disorder in the clinical sense of the term, but thought errors that enable the development of assumptions, beliefs, opinions, relationships and behaviors that could harm the person himself or others. Cognitive distortions are based on imprecise, partial, or irrational emotions and ideas. They, in fact, "enable" the offender to know that what he is doing is wrong, but to continue doing so. Treatment helps the patient see when the changeover 
from thought to behavior occurs. For example, "I know that I will fail (thought), so I won't even bother going to class (behavior)." This entire work focuses on cognitive elements. It is clear that a different method of intervention is required when dealing with these difficulties in a population defined as suffering from cognitive disorders and a lack of concrete thought.

\section{Confrontation}

One of the most efficient tools in therapeutic work with adolescents who have committed sexual offenses is confrontation, both in evaluating risks and needs (Etgar, 1997) and in treatment. But here we must ask to what extent should we engage in confrontation between the adolescents and their problematic behaviors, while due to their difficult childhood experiences they are already regarding criticism and confrontation from their therapists as an aggressive response to them. This tool in working with adolescents suffering from cognitive disorders is not only inefficient, but can even harm them and achieve the opposite effects among those who were criticized and "confronted" over their failures.

\section{Boundaries}

The topic of boundaries is crucial in treating adolescent offenders who have crossed all possible boundaries - moral, legal, human - and have actually penetrated the private space of another person. Treating each case of adolescents who have committed sexual offenses is individual in terms of the psychopathology that underlies their difficulty in perceiving personal boundaries and, as stated above, the disorder in their sense of empathy. But it is clear, on the other hand, that all adolescents breached interpersonal, legal and moral boundaries and it is vital to deal with this issue within the treatment process. Even so, one cannot ignore the difficulty to cope with the regulation of inappropriate behaviors that are typical to this specific population and the question to what extent one should be accepting or place clear boundaries that are crucial to the adolescent offenders.

\section{Attention and Concentration}

There are adolescents suffering from attention and concentration deficits, who do not belong to the population of offenders. There are adolescents who have difficulty concentrating throughout the meetings, due to emotional reasons (they feel threatened or fear the need to talk about the offense or anxiety in "remembering" what happened), but in regard to this population, some have severe difficulty in focusing throughout the meeting, because of attention and concentration deficits, that combine with a low level of intelligence. 


\section{Inadequate Ability to Internalize}

Many adolescents suffering from cognitive disorders experience difficulties in internalizing. Occasionally they "mindlessly repeat" the rules of what's permitted and what's prohibited, and therapeutic content, but most knowledge is not internalized and does not bring about a change of dangerous behavior. To be noted that this classification is not unique to this population, but in this context, we are witnessing a greater number of adolescents with inadequate abilities to internalize.

\section{Difficulty in Group Cohesion Deriving, Among Others, From Difficulties in Expressing Empathy}

The feeling is that every adolescent is concerned with his own interests, occupied with personal survival vis-à-vis the threatening situation of group participation. The threat of exposure, ridicule, insult, feeling different and an outsider, that was the fate of these adolescents in class or in other social peer groups over the years, is so great that it might overpower the huge advantages of participating in a therapy group. This is the situation also regarding the inadequate ability that creates difficulties in forming interpersonal relationships.

\section{Risk Level}

To be emphasized that this population is generally categorized as high risk for several reasons. First, the adolescents commonly suffer from difficulties in regulating impulses and emotions - and in many cases the crimes they committed were greatly impulsive and almost all lacked planning and contrivance, which are cardinal stages in the sexual offender's traditional progress within the Sexual Cycle. Second, we have seen that the actual conversation on sexuality (healthy or offensive) has occasionally caused high level sexual flooding, which is not typical to adolescents who do not belong to this population. In addition, the cognitive difficulties affected and, in many cases, also harmed the ability to make choices, reach decisions, understand cause and effect, social and logical understanding, moral inhibitions, ability to display empathy - all these are significant elements affecting the risk level. Finally, due to their low self-value and many years of feeling "different," these adolescents suffer from emotional limitation. In other words, not only do they suffer from an organic difficulty in their judgment and process of decision making, which are impulsive and narrow, there are also emotional elements (for them and their peers) that are not taken into account and do not exist in their system of considerations, when making a decision. The fact that for many years they were victims of humiliation, insult or abuse by the society, as well as suffering social rejection, caused them social regression, egocentric-survival limitation, to their primary concrete needs, which in turn developed low levels of empathy and moral values. The result of this 
is that there is no external or internal element that regulates inappropriate or abusive behavior towards others.

\section{Group Work}

One of the most accepted methods of treating adolescents who have committed sexual offenses is group work (Cunningham \& MacFarlane, 1991; Etgar, 1996a; Knopp, 1987). Due to the importance of this method for sexual offenders, we will give it special mention, while focusing on the population of adolescent who have committed sexual offenses with cognitive disorders, as described above.

Professional literature discusses the advantages of the group as a therapeutic tool, especially for adolescents, while emphasizing, among others, the importance of peer groups at this age, the need for a framework and a sense of belonging. This group's ability to develop unity provides this need. The group work also has advantages directly connected to the group of adolescent who have committed sexual offenses, such as coping with issues of shame and "secrecy," or content fields that are emphasized in the group work, which are connected to offensive sexual behavior (Etgar, 1999, p. 219). But, as stated above, it was found that the most efficient therapeutic method with sexual offenders is actually not suitable for adolescents suffering from cognitive disorders. Firstly, because the group therapy method for adolescent who have committed sexual offenses requires a normative level of intelligence and reasonable ability for abstraction. In addition, some of the work involves cognitive "psychoeducational" learning, such as what is sex by consent, the Sexual Cycle, etc., as well as a discussion on the wide range of abstract topics, such as "boundary," "choice," "responsibility," etc. In addition, the group is based on verbal abilities and, as stated above, the population of adolescents suffering from cognitive disorders is classified by low level labial-verbal abilities. Also, we must mention that adolescents suffering from cognitive disorders fear "others," because of their life experiences. They have no understanding of mutual help, listening, friendship, needing the assistance of one's peer group, but rather that of humiliation and rejection by "others."

\section{Belonging}

From all that described above, the adolescents suffering from cognitive disorders feel a sense of alienation. They do not feel that they belong in this world or society and specifically their participation in a unique therapeutic group with "others like them" will give them a sense of partnership and belonging that might be beneficial to them. 


\section{Variance and Needy}

From a young age these adolescents feel different and lonely, mainly because of their continued experiences of failure. Group therapy can help alleviate these feelings, as well as the sense of great neediness. Due to these factors, adolescents suffering from cognitive disorders will have difficulty, and might not even be able to integrate into existing treatment groups that use the classic method. It was clear that several adjustments must be made, to help them benefit from this method of treatment.

\section{Solutions, Responses and Adaptations to the Difficulties of Adolescents Suffering From Cognitive Disorders}

One of the terms suggested by O'Callaghan (2006) to use in the therapeutic process is the transfer from "from an old life to a new life," which includes the following elements:

- An understanding that the change is a continuous process enabling the previous behavior of the "old life" not to awaken a sense of despair, but to create an opportunity to promote the positive goals and learn how to cope with negative and dangerous behaviors.

- By using the term "new life" and not "the new me," there is recognition and acceptance that others, such as family members and therapists, play a significant role in molding the adolescent's quality of life and must contribute to the process of change.

- A method to negatively tag the behaviors in the "old life" and positively tag those in the "new life."

- Monitoring the adolescent's progress in the program.

- Tagging the ideas and behaviors with which the adolescent must cope as reflecting his old life or his new life. By defining these stages as old life and new life, it is possible to continue attributing each of the patient's notions, actions or thoughts and categorize them to the new ideal me, which I aspire to reach, or to the old me.

Further on we will detail, in short, several elements from the work on the Sexual Cycle and mention a number of responses we found to be effective during our experiences over many years with this population.

\section{Emotions}

Adolescent boys have difficulty expressing a variety of emotions, and generally erupt in anger or sadness. Therefore, as a contributing factor to the work on emotional events at the start of the cycle, we will focus on the acquaintance and identification of physiological sensations affiliated with anger, frustration, and helplessness. In other words, we spoke about physical sensations and the adolescents connected to that very well. We 
asked them to describe what happened to them after the event and received diverse responses, such as: my hair stood on end, pressure in my chest, heat, electricity, etc. In the second stage we gave the physical sensation a name and connected it to a behavior, for example, when they were "irritable," they behaved in a certain way, such as banging their head on the wall, hitting, breaking the telephone, or sexually abusing someone. Getting to know the body's physiological responses and discussing the relating behaviors helped the adolescents identify and recognize themselves and their physiological and emotional responses, as well as later understanding the alternatives to their behavior and to their expression of anger.

\section{Thoughts}

Frequently these adolescents could not report on any intermediate thoughts between the physiological or emotional arousal and the behavioral expression. Both the self-reporting and the objective description (for example, found in the statement of claim) showed a high level of impulsivity during the offense. Even when there was a cognitive process before the offense, it was low, almost lacking any planning or manipulation, doubts or moral hesitation. The main purpose was to arouse their hesitation, to strengthen the ability to mentalization and thus to raise their self-esteem, level of distinction, and self-value; to show that there are several possible thoughts about the same event that exist concurrently. One of the adolescents described this at the end of the therapeutic process as a scale that has positive and negative thoughts and, in the end, the one that balances them will affect the response/behavior. In specific refence to the abstract terms of "errors in thought," it is often spoken about the "deceiving brain" or "the head isn't working well," etc. It is obvious that just like any treatment with adolescents, it has been emphasized that this is a universal phenomenon that happens to everyone.

\section{A Relationship Between Cause and Effect}

One of the greatest difficulties was an understanding of the relationship between cause and effect. Some adolescents understood this after we gave them several examples, while others had greater difficulty and it took them longer to understand.

\section{Emotional Regulation}

During this work there arose a need to provide tools for emotional regulation. Most of the adolescents described that punching a wall and smoking cigarettes is relaxing. Together we expanded the possibilities of how to relax (for example, we performed exercises of stretching and relaxing muscles and breathing). During the meetings the adolescents experienced success in regulating their emotions and even told about positive experiences in their daily lives. 


\section{Information About Sexuality}

The information that these adolescents have about sexuality is mainly from pornography, which majorly distorts facts. In addition, they lack a lot of information on sexual development and adolescence. Therefore, when we feel that they are lacking information to understand and cope with situations, we explain and check how this connects to their world and if it clashes with other facts they experienced or knew. To be mentioned that this matter was brought up also in meetings with adolescents who do not suffer from cognitive disorders. But, here the knowledge of "healthy sexuality" was exceptionally sparse and, on the other hand, the "information" they had from viewing pornography, without any intervention, was often perceived as total truth. This is how children are conceived and this is how a husband treats his wife. It is important to note that because of their low self-image, experiences of rejection and humiliation that they experienced throughout their lives, the process of "adopting" what they viewed in pornographic movies was extremely quick and efficient. First, because it was a supposedly effective response both to the need to improve their self and gender value (like with "normative" boys) and because it gives an immediate response to their aggressive and sexual impulses. For example, Yitzchak hurt girls on the bus, when he suddenly grabbed their chest. From his perspective, this action was a way of "letting her know" that he was interested in having sexual relations with her and he could not understand why she did not respond in the same way that the girl in the pornographic movie, which he had seen, responded.

We must mention that when working with this population the Sexual Cycle is more difficult to understand as it has greater abstract elements. In more advanced meetings, we spoke about the model and drew the Cycle together with the adolescents. While doing this we explained the various stages, as some of their terms were not easily understood. We started by describing the offense; the adolescents cooperated and described their actions. Then we spoke about the period of time before the offense, what life was like before, were there significant events, what emotions they were coping with, etc. Throughout the process we tried to understand the existing distortions of thought. This is the changeover between the "old life" and the "new life." There were patients who had difficulty in understanding the Cycle and especially in applying it to their daily lives. We continued contact with them and practiced the process between events - physiological-emotional-thought arousal - action and therapeutic work on emotional regulation, as stated above. Many patients succeeded (after much repetition) to understand the Sexual Cycle and even to apply it to their daily lives. in this regard there is much similarity to working with patients who do not suffer from cognitive disorders, but the process is extremely slow and involves a lot of demonstration and repetition.

To be noted that some of the adolescents (those with a high level of intelligence and greater ability for abstract thought) actually connected to, and applied, the so-called Efrat Model in their daily lives. The Efrat Model [based on a Hebrew acronym] is a cog- 
nitive-behavioral model that is based on "engagement, assessment, insight, reorientation" and places the person's understanding of events in their lives in a pivotal position.

\section{Integrating Various Working Methods, According to the Characteristics of This Population}

Here we must mention that, in order to intensify the knowledge and set up the first groups, we held orientation meetings with the therapeutic team at the Shekel Center in Jerusalem, a nonprofit organization that provides community services for people with special needs. These meetings emphasized the special needs of these adolescents suffering from cognitive disorders and the methods recommended to use when working with them. Following are several points relating to the working methods and frameworks we used for this population:

- A smaller group - while groups of adolescents who have committed sexual offenses generally comprise up to eight adolescents, we have five or six participants in each group.

- Psycho-diagnostic evaluations as a precondition for this kind of treatment - the evaluation indicates a lower cognitive level (lower or borderline intelligence, but not to a mental-developmental disability) and checks if they are learning in a special-ed environment.

- Duration of meetings is shorter - meetings are generally 1.5 hours. Due to difficulties in the adolescents' coordination, we planned the first fifteen minutes for organization: food, drink, bathroom, etc.

- We devised an adapted and abridged contract for the group in which we stated the rules in a simple language with short sentences. We worked on the contract over several sessions, while thoroughly explaining each section and giving examples from the adolescents' lives.

- Speaking in a clear and simple language and stopping frequently to clarify terms, while making sure that all participants understand specific sentences or statements. Many repetitions of sentences or statements in other, simpler, words, both during the sessions and between sessions. The repetition of terms, topics and content used in the sessions is an important technique in dealing with these groups. O'Callaghan (2006, p. 336) emphasizes this in reference to a specific exercise and says that by using repetition in the group, on the one hand, and having the other group participants view the exercise and perform the same, on the other hand, turns it into a recognized method that enables the adolescent to describe his thoughts and emotions on the topic.

- The increased number of positive feedbacks on every positive behavior, a statement showing understanding or the sharing of significant difficulties in the group, or any display of empathy that might help another friend in the group. 
- Tangibility - having the therapists understand that the work is concrete and limited to the immediate situation, without the ability to see the expanded background or think abstractly.

- Expanded use of auxiliary tools, such as an emotions board, writing on a board, which helps focus attention.

- Use of humor.

- A supporting environment and contact with the parents, social workers in offices, parole officers or social workers in hostels, is much closer.

- The interview with the adolescents coping with cognitive disorders requires skills, as there is difficulty in estimating their level of motivation and danger. Emphasis during interviews must be put on the ability to talk about the offense and examine if the adolescent actually admits to having committed the crime. This must be emphasized to the counselors who must undergo special training before the interviews.

- A supportive environment/significant figures - the process is emotionally and sexually flooding and one must examine the environment and the strength within it to ensure support in the process of minimizing risks. At the same time, one must understand the danger posed by this adolescent also to the environment in which he lives. This is in combination with the limiting conditions.

- "From old life to new life" - perceiving the situation through these "glasses," and bringing the facts to these adolescents also in different versions, can be a milestone in the comprehensive perspective of our work. We mentioned this above, and here we will emphasize and specify: the entire issue of a supportive environment and having the family join the process is part of building a "new life." It is important to mention that due to the risk factors and cognitive difficulties, the matter of protectiveness and family/community support is significantly more important. Therefore, great emphasis must be put on the supportive environment in an attempt, as much as possible, to create a full agenda and prevent "idleness." This is also a response, albeit partial, to the emotional limitation that characterizes many adolescents with cognitive disorders.

\section{And What About the Therapists?}

While remaining loyal to our belief that the therapist holds a very important place in the therapeutic process and that we have the obligation to protect him (Etgar \& Davidson-Arad, 1998), we would like to say a few words on behalf of those treating this population. The therapists expressed their doubts and difficulties in the same issues (as mentioned above) that are difficult for the patients. When focusing on the patient, the question was "how to cope with the difficulties of inappropriate behavior/s that are typical of these adolescents," "to what extent would I be accepting of an adolescent" or "how do I place boundaries for this adolescent?" while during counseling the same issue 
was perceived as "how do I cope with these behaviors," and "how do I put boundaries in a different way from what I am used to in regular treatments?"

We have seen the same situation in regard to the question of friction. How much can we confront these adolescents with their problematic behaviors, while, due to their difficult childhood, they regard any criticism or friction from the therapist as a violent approach towards them. In regard to the difficulty in internalization - it was interesting to see the difference in respect to these therapists. One can assume that when dealing with these patients, empathy is a most effective therapeutic tool available to the therapists. The therapists' approach of empathy and care is what will initiate the change. In addition to this, the therapist also has to deal with empathy towards someone who committed a sex offense.

Many times, while working with diverse populations (not necessarily with cognitive disorders) we find that therapists report adolescents "reciting," but not really emotionally connected, to what he is saying. Occasionally this annoys the therapists. When dealing with adolescents suffering from cognitive disorders, the therapists were much more patient and spoke about "difficulty in internalization."

We have seen the same pattern also in regard to errors in thought, working on the cycle, attention and concentration disorders, as well as the "discovery" that, despite a common denominator of cognitive difficulties, there is variance on the level of difficulties, verbal expression, internalization and cognition, a matter that sometimes makes adapting the content to the entire group more difficult. As mentioned by the group counselor, "also they are adolescents like all adolescents, different from each other ...".

At the outset of treatment, therapists have occasionally expressed feelings that everyone is "different," "strange," "not understanding," but gradually, as therapy progressed and with the development in our work, the situation changed - "it is a challenge to work with them," "there is a change in discipline, the therapeutic approach is different than what we know. It is interesting and challenging."

The literature (Etgar, 1996b) shows a discussion on the parallel processes in groups of adolescents who have committed sexual offenses, and their counselors. Also, when the patients suffer from cognitive disorders, the same phenomenon is expressed. The therapists spoke about many expressions of ambivalence, both with the patients and with themselves: On the one hand the adolescents come regularly for treatment and put in an effort to make a change and, on the other hand, it is difficult to create trust in the ability to change or in the environment. This is the same with us the therapists. On the one hand there is a sense of repetition and sometimes a feeling of being immersed in treatment, but, on the other hand, there is a sense and reality of treatment which is felt as critical and vital, based on maintenance treatment and constant support, which is needed for these adolescents on a regular basis.

The counselors, in a manner more emphasized that with other groups, were very concerned with questions about the time after treatment. "What will be afterwards?" 
What supportive and maintenance treatment will they continue to receive? And what about the protection programs? From the therapists' statements and a collection of contents from the counseling sessions, we saw that they expressed an understanding of "necessity," from their perspective, to be softer and more accepting. In addition, they expressed great difficulty in "meeting" with their own aggression.

We believe that this is also due, partially, to the working frameworks which were more confrontational and the therapist had some legitimacy to accept and even to express, in some cases, their own aggressiveness, disgust from the actions and the anger and hostility that these actions aroused. On the other hand, in a working method that is almost devoid of confrontation, it is much harder for the therapist to express his own aggression and this has unique significance for the therapists.

\section{Sum mary}

In the current article we threw some light on a unique group of adolescents who have committed sexual offenses, who suffer from cognitive disorders. Throughout the article we related to the characteristics and difficulties that are unique to this population and presented the solutions and issues that are especially important to be addressed when dealing with these adolescents. This population has low cognitive levels resulting from organic developmental difficulties. Therefore, for them, mental tasks involve a greater effort than what is required from an adolescent without difficulties, and when they come to us for treatment, they are already "carrying on their backs" ongoing experiences of anxiety, frustration, anger, failure, disappointment and pain from coping with basic growth and development efforts.

The purpose of therapy is to provide these adolescents with psychotherapeutic help, which is adapted to their abilities, in order to decrease their chances of recidivism. Throughout this paper we found that the most effective therapeutic model for sex offenders, i.e., group work combined with cognitive elements, is not appropriate for these adolescents suffering from disorders in this field, in light of their difficulty with abstraction, internalization, organization, etc. As a result, we have constructed special groups for this population, which give reference also to the special needs of adolescents suffering from cognitive disorders and adapts the recommended working methods with them, such as: a limited number of participants in each group, an extended use of auxiliary tools, a group where the boundaries are more lenient, the use of a simpler language, adapted contract and time for organization, as well as the entire current working process. We found that it was not effective to work with a group of adolescents suffering from cognitive disorders as with any other group and we must make required adjustments by virtue of the participants' specific characteristics.

One of the issues raised around the therapeutic process is the transfer from the "old life" to the "new life." In other words, an understanding that the change is an 
ongoing process, which minimized the sense of despair vis-à-vis ineffective behaviors ("the old life"), while simultaneously creating the opportunity to learn how to cope with difficulties and how to create a protective environment and recruit the family, who are a significant part of building the "new life" and minimizing the danger of recidivism. Our purpose in the current article was to share our experience and insights from our work with adolescents who have committed sexual offenses suffering from cognitive disorders. We hope that our experience will be a basis for continued learning and development of additional methods and techniques that can help in our work with this population.

Funding: The authors have no funding to report.

Competing Interests: The authors have declared that no competing interests exist.

Acknowledgments: The authors have no support to report.

\section{References}

Brannon, J. M., \& Troyer, R. (1995). Adolescent sex offenders: Investigating adult commitment-rates four years later. International fournal of Offender Therapy and Comparative Criminology, 39, 317-326. https://doi.org/10.1177/0306624X9503900404

Craig, L. A. (2017). Working with sexual offenders with intellectual developmental disabilities. In D. T. Wilcox, M. L. Donathy, R. Gray, \& C. Baim (Eds.), Working with sex offenders: A guide for practitioners (pp. 136-161). Abingdon, United Kingdom: Routledge.

Cunningham, C., \& MacFarlane, K. (1991). When children molest children. Brandon, VT, USA: Safer Society Press.

Etgar, T. (1996a). Sex offenses among adolescents: The forum for children and adolescents at the Bob Schappel School of Social Work, Tel Aviv University and ELEM. Tel Aviv, Israel: Ramot Press.

Etgar, T. (1996b). Parallel processes in a training and supervision group for counselors working with adolescent sex offenders. Social Work with Groups, 19, 57-69.

https://doi.org/10.1300/J009v19n03_05

Etgar, T. (1997). Diagnosing adolescent sex offenders. Tel Aviv, Israel: ELEM, Nonprofit for Youth at Risk and in Distress and the Bob Schappel School of Social Work, Tel Aviv University and the Israel National Council for the Child, the Center for Research and Public Policy.

Etgar, T. (1999). Group therapy for adolescent sex offenders. Society and Welfare, 2, 215-234.

Etgar, T. (2012). Life and death are in the power of the tongue. Sexual Offender Treatment, 7, 1-11. http://www.sexual-offender-treatment.org/106.html

Etgar, T., \& Davidson-Arad, B. (1998). The therapist/assessor of adolescent sex offenders: Issues, difficulties and dilemmas. Tel Aviv, Israel: Ramot Press.

Etgar, T., \& Neder, N. (2008). "Sewn from the Same Fabric, by the Patient's Measurement" - Individual reference to principles of treatment in adolescent sex offenders: From risk to opportunity of 
therapeutic interventions in youth by law and in minors in distress. Brandon, VT, USA: Safer Society Press.

Fisher-Caspi, M., \& Vangruber-Neumann, G. (2010). And a wolf shall live with a lamb - With us as well? [Electronic version]. Retrieved from https://www.hebpsy.net/articles.asp?id=2532

Knopp, F. H. (1987). The youth sex offender: The rational and goals of early intervention and treatment. Brandon, VT, USA: Safer Society Press.

Lane, S. (1991). The sexual abuse cycle. In G. Ryan \& S. Lane (Eds.), Juvenile sexual offending (pp. 103-141). Lexington, MA, USA: Lexington Press.

Lindsay, W. R., Neilson, C. Q., Morrison, F., \& Smith, A. H. (2001). A comparison of physical and sexual abuse histories of sexual and non sex offenders with intellectual disability. Child Abuse \& Neglect, 25, 989-995. https://doi.org/10.1016/S0145-2134(01)00251-4

Maletzky, B., \& Steinhauser, C. (2002). A 25-year follow-up of cognitive-behavioral therapy with 7,275 sex offenders. Behavior Modification, 26, 123-147.

https://doi.org/10.1177/0145445502262001

Marotta, P. L. (2017). A systematic review of behavioral health interventions for sex offenders with intellectual disabilities. Sexual Abuse, 29, 148-185. https://doi.org/10.1177/1079063215569546

O'Callaghan, D. (2006). Group treatment of young people with intellectual impairment who sexually harm. In R. E. Longo \& D. S. Prescott (Eds.), Current perspectives: Working with sexually aggressive youth and youth with sexual behavior problems (pp. 325-348). Holyoke, MA, USA: Neari Press.

Ofek, A. (2006). Group treatment program for adolescent sex offenders. Ramat HaSharon, Israel: Ofek Institute for Management and Research.

Schladale, J. (2006). Family matters: The importance of engaging families in treatment with youth who have caused sexual harm. In R. E. Longo \& D. S. Prescott (Eds.), Current perspectives: Working with sexually aggressive youth and youth with sexual behavior problems (pp. 493-514). Holyoke, MA, USA: Neari Press.

Thibaut, F., Bradford, J. M. W., Briken, P., De La Barra, F., Häßler, F., Cosyns, P., \& WFSBP Task Force on Sexual Disorders. (2016). The World Federation of Societies of Biological Psychiatry (WFSBP) guidelines for the treatment of adolescent sexual offenders with paraphilic disorders. The World fournal of Biological Psychiatry, 17, 2-38. https://doi.org/10.3109/15622975.2015.1085598

Thompson, D., \& Brown, H. (1998). Response-ability: Working with men with learning disabilities who have difficult or abusive sexual behaviours. Brighton, United Kingdom: Pavilion.

Zankman, S., \& Bonomo, J. (2004). Working with parents to reduce juvenile sex offender recidivism. Journal of Child Sexual Abuse, 13, 139-156. https://doi.org/10.1300/J070v13n03_08 


\section{IATSO}

International Association for the

Treatment of Sexual Offenders

Sexual Offending: Theory, Research, and Prevention is the official journal of the International Association for the Treatment of Sexual Offenders (IATSO).

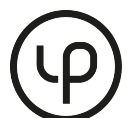

leibniz-psychology.org

PsychOpen GOLD is a publishing service by Leibniz Institute for Psychology Information (ZPID), Germany. 